\title{
Understanding the impact of Time-lag Full-waveform Inversion on dual-azimuth data
}

\author{
Erick Tomaz, Abraham Rodriguez, Yan Borges, Gregory Culianez, CGG
}

Copyright 2021, SBGf - Sociedade Brasileira de Geofísica

This paper was prepared for presentation during the $17^{\text {th }}$ International Congress of the Brazilian Geophysical Society held in Rio de Janeiro, Brazil, 8-11 November 2021.

Contents of this paper were reviewed by the Technical Committee of the $17^{\text {th }}$ International Congress of the Brazilian Geophysical Society and do not necessarily represent any position of the SBGf, its officers or members. Electronic reproduction or storage of any part of this paper for commercial purposes without the written consent of the Brazilian Geophysical Society is prohibited.

\section{Abstract}

Lack of low-frequency content, limited offsets, and narrow azimuth (NAZ) distribution in seismic data are three wellknown drawbacks to streamer acquisitions, compromising the chances of success for full-waveform inversion (FWI), particularly for pre-salt targets. Broadband data has emerged as a cost-effective way of obtaining better low frequencies, and long cables are deployed to improve diving wave penetration. In addition, Time-lag FWI (TLFWI) technology, supported by advances in computational power, have allowed for higher quality inversions from NAZ acquisitions. However, lack of illumination still poses a considerable obstacle to imaging deep target areas. We present a study of TLFWI applied to a dual-azimuth (DAZ) dataset from deep-water offshore Brazil, where we leverage the benefits of longer streamers and an additional azimuth to obtain an improved pre-salt image in the context of complex geological formations.

\section{Introduction}

Subsurface imaging quality is directly related to the quality of the field data preprocessing, the fidelity of the velocity model, and the ability of Reverse Time Migration (RTM) to handle complex wavepaths. Each of these factors has a considerable impact on the quality of the final image. However, they are constrained by the seismic data acquisition and its intrinsic limitations. As such, acquisition design is a key element in the success of the final imaging.

Nevertheless, new acquisitions are often planned based on limited understanding of the geology of the area, relying on older datasets processed with legacy technology such as time migration. Consequently, acquisition directions are often not optimal, particularly in areas of complex geology like Santos Basin, where overburden, salt thickness, salt shape, and pre-salt structures pose significant challenges for imaging.

As a result, NAZ velocity model building and imaging are hindered, especially for deep targets below complex structures. One way to overcome these limitations is to reassess shooting direction and cable length prior to a second acquisition campaign, and base acquisition plans on more reliable interpretation extracted from more recent 3D NAZ images.

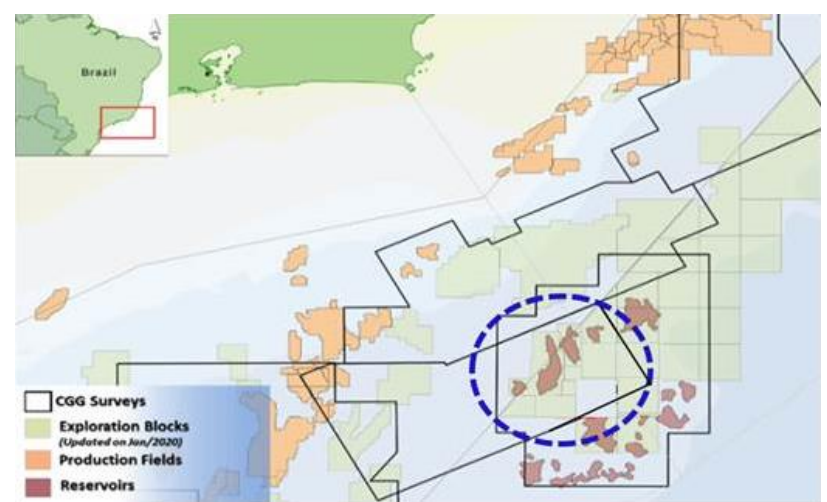

Figure 1. Location map of both surveys used in the study.

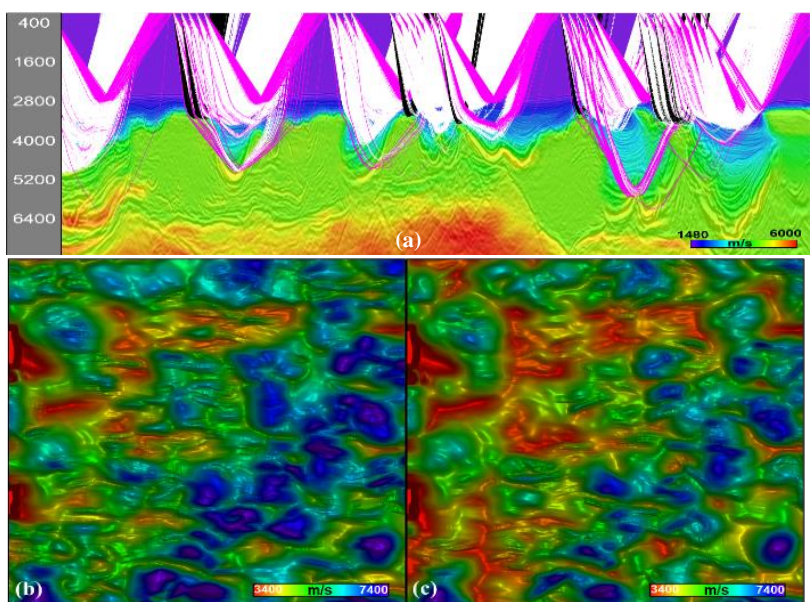

Figure 2. Diving rays: (a) offset 0 to $4000 \mathrm{~m}$ (black line), 4001 to $8000 \mathrm{~m}$ (white line), 8001 to $10000 \mathrm{~m}$ (pink line); (b) maximum depth penetration map with $10 \mathrm{~km}$ offset; (c) maximum depth penetration with $8 \mathrm{~km}$ offset. 


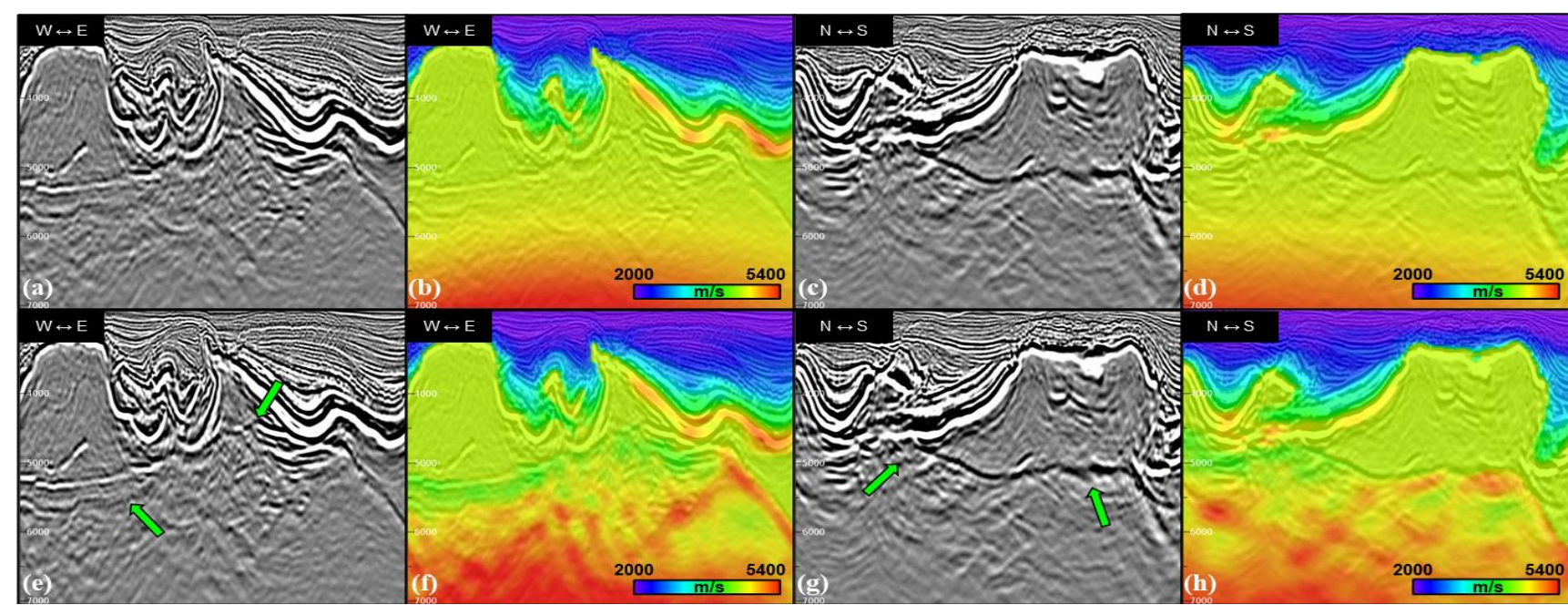

Figure 3. Input $20 \mathrm{~Hz}$ RTM migration using Survey A data (a) inline and (c) crossline. Input velocity model (b) inline and (d) crossline. $20 \mathrm{~Hz}$ RTM using Survey A data (e) inline and (g) crossline. Survey A TLFWI: (f) inline and (h) crossline.

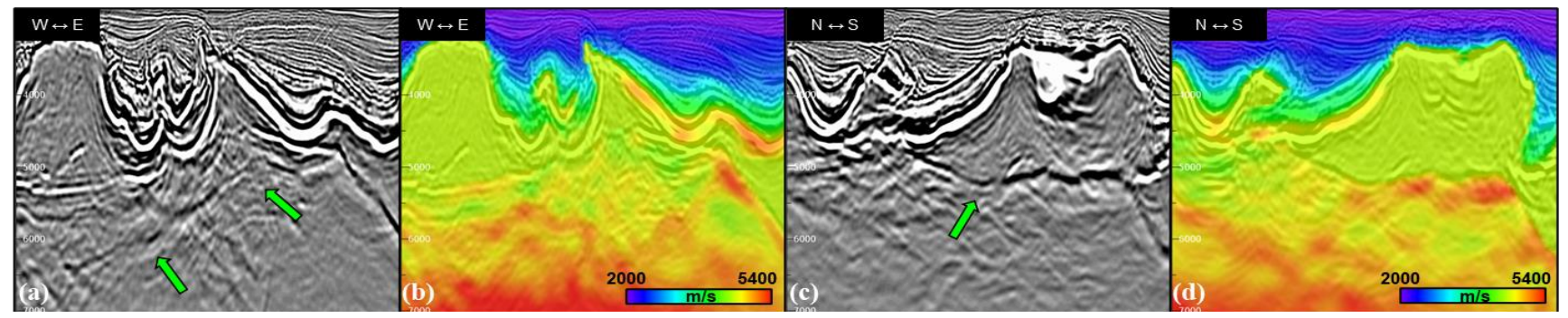

Figure 4. $20 \mathrm{~Hz}$ RTM using Survey B data (a) inline and (c) crossline. Survey B TLFWI (b) inline and (d) crossline.

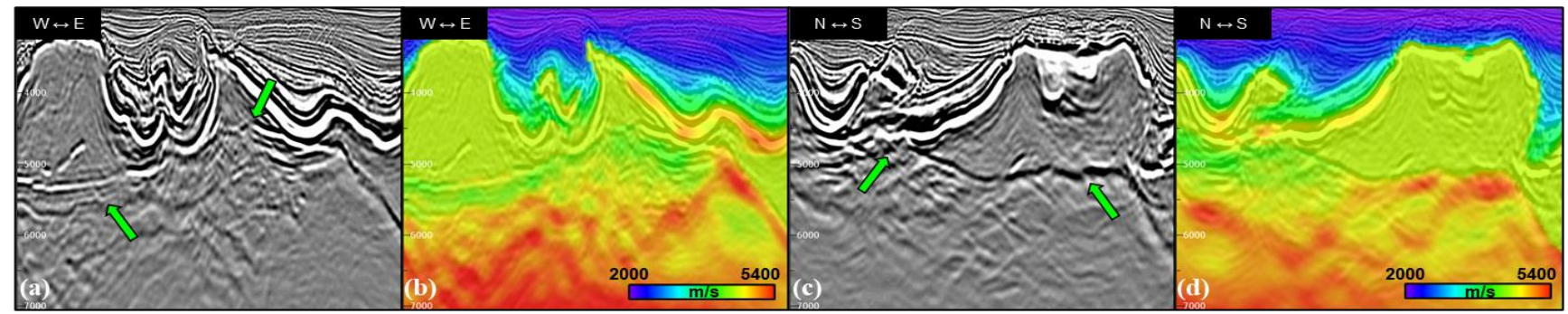

Figure 5. $20 \mathrm{~Hz}$ RTM using Survey A data and DAZ TLFWI model (a) inline and (c) crossline. DAZ TLFWI (b) inline and (d) crossline.

The second NAZ acquisition should then complement the previous one to provide a more reliable image and velocity model, improving the overall stability of TLFWI (Zhang et al., 2018). This combination also helps minimize the problem of weak spatial distribution of NAZ surveys, ultimately leading to an enhanced velocity model.

Since the quality of the subsurface image is highly related to the complexity of the overburden, it is very common in NAZ projects, due to the poor azimuth distribution, to have areas where we lack information, generating errors in the image. Most importantly, the cause of these issues is not clear, e.g., velocity model errors, illumination issues, etc. We will demonstrate how a second azimuth, when smartly planned, can reduce some of these uncertainties and thus give more confidence in prospect studies and well positioning plans.

Here, we present TLFWI results produced using DAZ data from the deep-water region of the Santos Basin. The two surveys, Survey B and Survey A (Figure 1), both used streamers with depths varying from $10 \mathrm{~m}$ to $30 \mathrm{~m}$. Survey $B$ was acquired with an $8 \mathrm{~km}$ maximum offset and azimuth $\mathrm{N} 152.5^{\circ}$. Survey $\mathrm{A}$ is the most recent acquisition, finished in December 2020, with longer streamers $(10 \mathrm{~km})$ and azimuth $\mathrm{N} 89.6^{\circ}$. A nominal streamer separation of $100 \mathrm{~m}$ is common to both acquisitions.

\section{Method}

One of the key elements for successful FWI is the quality of the input data, particularly at the low frequencies. Because of this, dedicated denoise processing was applied to improve the S/N prior to $\mathrm{FWI}$, and phase quality control 


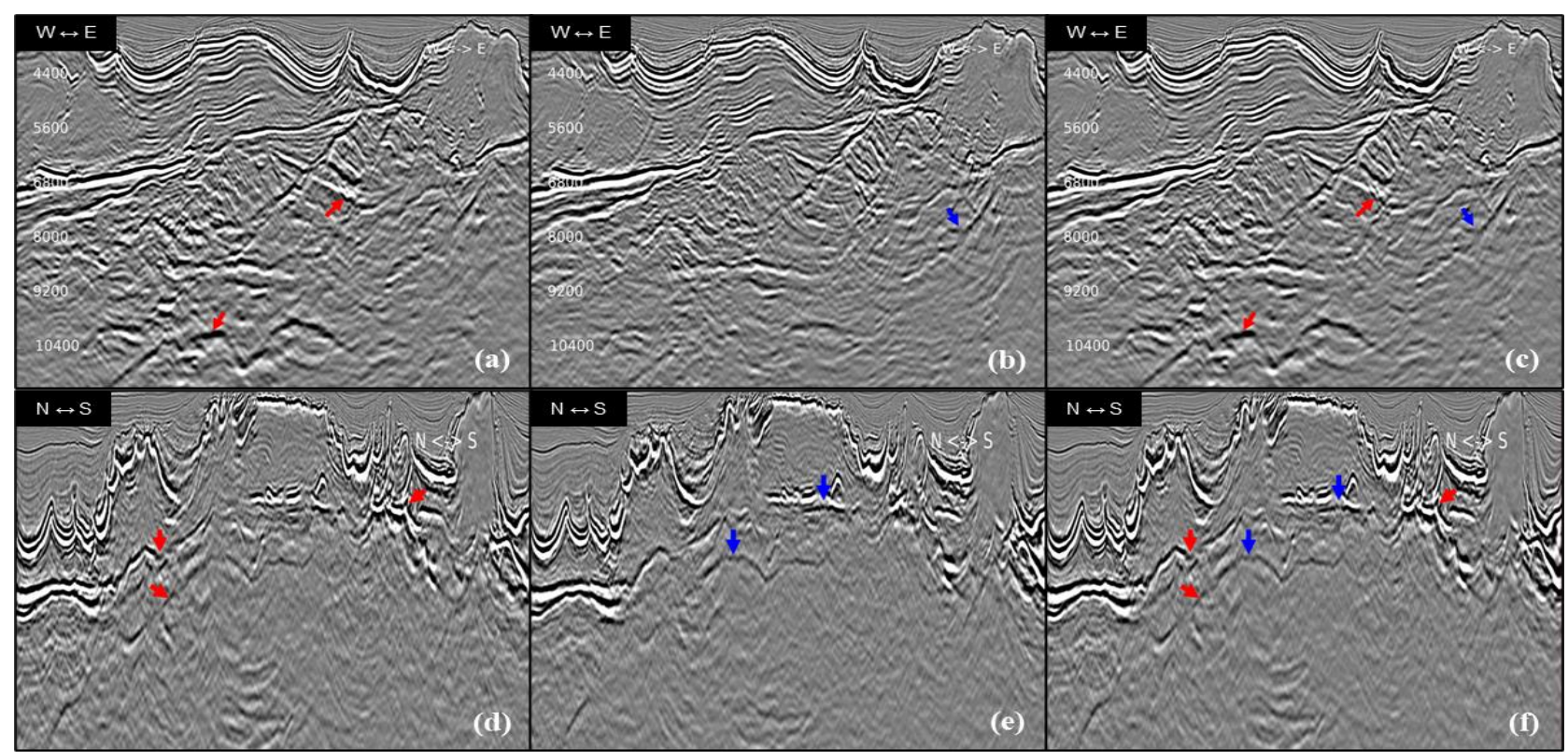

Figure 6. $20 \mathrm{~Hz}$ RTM using dataset and velocity model for: Survey A (a) inline, (d) crossline; Survey B (b) inline, (e) crossline; and Dual-Azimuth (c) inline, (f) crossline. The DAZ image was built using the DAZ velocity model and MAZ stack technique.

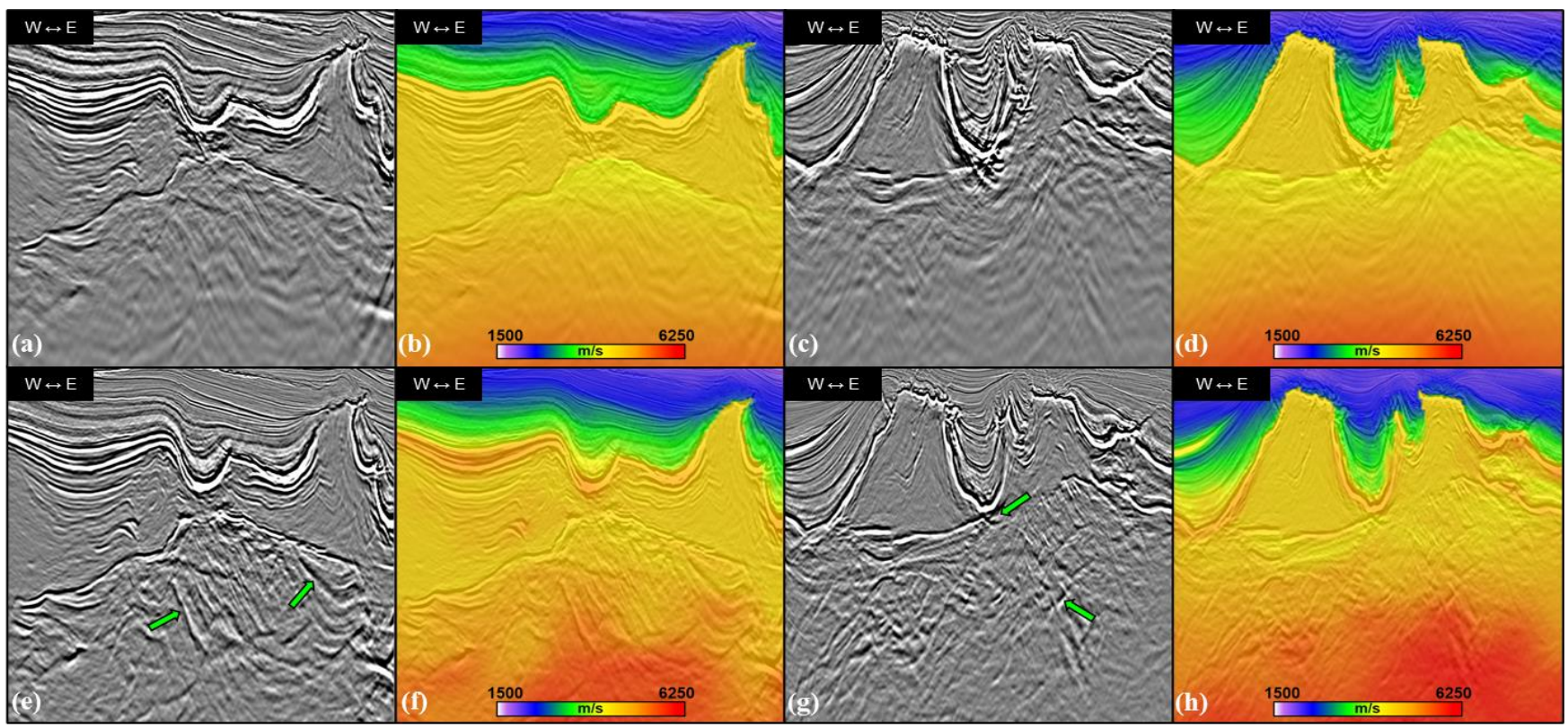

Figure 7. $45 \mathrm{~Hz}$ RTM using dataset and velocity model for: Legacy NAZ processing with Survey B (a), (b), (c), (d); and Dual-Azimuth processing with Survey A \& Survey B (e), (f), (g), (h).

was used to define the most useful and reliable starting frequency for inversion.

As previously mentioned, having two surveys acquired in different directions can potentially alleviate illumination issues in some areas, especially considering that the newest acquisition provides additional offset information. However, we can still expect some limitations due to the lack of diving waves, as most of the recorded information is related to the reflections. In order to mitigate this, we use the full wavefield data including primary reflections, diving waves, and multiples in the inversion.
A smoothed version of the Survey B legacy velocity was used as the starting point for the current TLFWI. It is important to mention that this legacy velocity was built using only Survey B data. Shots from both surveys were then used simultaneously for the TLFWI inversion.

In order to understand the contribution of each survey to TLFWI, three inversions were computed: using only the recent $10 \mathrm{~km}$ offset survey; using only the $8 \mathrm{~km}$ offset survey; and finally using both.

With the velocity model from DAZ TLFWI, a RTM for each survey was performed and then images were combined 
using a Multi-azimuth (MAZ) stack approach that aims to take the best of each acquisition direction by distributing weights based on relative amplitude and coherence (Hung \& Yin, 2012)

In addition, we performed RTM using each dataset and its respective velocity model: Survey $A$ (longer offsets) with Survey A model; Survey B data with Survey B model; and finally both RTM images combined using the MAZ stack approach with DAZ model.

\section{Results}

Due to the nature of this case study, where the acquisitions differed in both offset and azimuth, it is difficult to isolate the effects of the longer offsets from the azimuthal differences. Therefore, to evaluate only the impact of the longer offset data for velocity model building, we performed ray tracing to obtain a diving ray depth penetration analysis.

As such, we can compare the penetration depth between maximum offsets of $8 \mathrm{~km}$ and $10 \mathrm{~km}$ using the Survey $A$ acquisition direction. The maximum penetration depth maps and an inline are shown in Figure 2. They demonstrate that these extra $2 \mathrm{~km}$ allowed deeper penetration of the diving waves and, as a consequence, a more stable inversion.

The post-salt section suffers less from illumination issues and is quite well resolved by both azimuths. The most significant changes on the image can be seen at the presalt level, which is coherently identified and imaged when inverting only Survey A data (Figure 3) or only Survey B data (Figure 4). There is a clear uplift compared to our starting model and although the velocity model trend looks similar between the two, we can highlight that velocity field definition in the high reflectivity compartments and around the fault planes is less accurate in the Survey B inversion.

It is possible to observe that the TLFWI inversion using both datasets (Error! Reference source not found.5) incorporates benefits from each azimuth contribution and provides better event definition, particularly for salt body boundaries. We note that the DAZ model image is superior, maintaining the enhancements seen of each NAZ model image.

This is corroborated by the improved imaging response on each separate RTM migration and their combined MAZ stack when using the DAZ velocity model (Error! Reference source not found.6). It is clear that the DAZ image benefits from the contribution from both datasets (blue and red arrows on Figure 6). In Figure 7, we can see a final comparison between the legacy NAZ processing using only Survey $B$ data against the $D A Z$ processing that includes the DAZ TLFWI and the MAZ stack approach, showing a significant improvement in the image at the presalt level.

\section{Discussion}

Combining different shooting directions allowed us to overcome some of the illumination issues. The two surveys complemented each other in areas where one or the other lacked information. Of course, this is highly related to the acquisition direction (Etgen and Regone, 1998), usually chosen based on the structural orientation, which in our area of study at the Santos Basin are predominantly N-S and NE-SW (Zalan, 2004; Zalan et al., 2011).

Considering the geological complexity of Santos Basin and the limited azimuthal information recorded by a NAZ acquisition, we have shown that a DAZ approach can help to improve the velocity model fidelity through TLFWI combined with MAZ stack algorithm.

Even though the DAZ process has improved the quality of the image, there are still some remaining challenges and uncertainties that will most likely only be minimized by a more robust acquisition with richer azimuthal coverage, such as Ocean Bottom Nodes (OBN) or Sparse-OBN (Xue et al., 2020).

\section{Conclusions}

Legacy narrow-azimuth towed-streamer data often suffers from poor diving wave penetration, lack of high-quality low frequencies, and inconsistent illumination. More modern acquisitions use longer offsets to improve diving wave penetration, and variable-depth streamers for better lowfrequency content. However, a lack of azimuthal information remains a challenge. We show that by leveraging two NAZ datasets to compose a DAZ dataset, we can obtain a velocity model that is superior to those obtained from the individual NAZ acquisitions. Our results support a conclusion that these improvements come from complementary information found in both datasets.

\section{Acknowledgments}

We thank CGG Multi-Client for permission to publish this work and Carlos Costa, Filipe Rudrigues, and Adel Khalil for their support.

\section{References}

HUNG, B., \& YIN, Y. 2012. Optimal stacking for multiazimuth pre-stack seismic data. 22nd International Geophysical Conference and Exhibition, 26-29 February 2012 - Brisbane, Australia.

ETGEN, J., and REGONE, C. 1998. Strike shooting, dip shooting wide patch shooting - Does pre-stack depth migration care? A model study: 68th Annual International Meeting, SEG, Expanded Abstracts, 66-69.

ZHANG, Z., MEI, J., LIN, F., HUANG, R., and WANG, P., 2018, Correcting for salt misinterpretation with fullwaveform inversion: SEG International Exposition and $88^{\text {th }}$ annual Meeting, 1143-1147.

ZALÁN, P. V. Evolução Fanerozóica das Bacias Sedimentares Brasileiras. Geologia do Continente Sulamericano - evolução da obra de Fernando Flávio Marques de Almeida. [São Paulo]: Ed. Becca, 2004. p.595-612. 
ZALÁN, P. V., SEVERINO, M. C.G, RIGOTI, C.A., MAGNAVITA, L.P., OLIVEIRA, J.A.B, \& VIANNA, A.R, 2011, An Entirely New 3D-View of the Crustal and Mantle Structure of a South Atlantic Passive Margin - Santos, Campos and Espírito Santo Basins, Brazil: AAPG ANNUAL CONVENTION AND EXHIBITION, Expanded abstract, p. 12.

XUE, Z., ZHANG, Z., LIN, F., MEI, J., HUANG, R. \& WANG, P., 2020, Full-waveform inversion for sparse OBN data: $90^{\text {th }}$ Annual International Meeting, SEG, Expanded Abstracts, 686-690. 